\begin{tabular}{l} 
PHILOSOPHICAL \\
TRANSACTIONS \\
$\begin{array}{l}\text { THE ROYAL } \\
\text { SOCIETY }\end{array}$ \\
\hline
\end{tabular}

\title{
Modelling aspects of cancer dynamics: a review
}

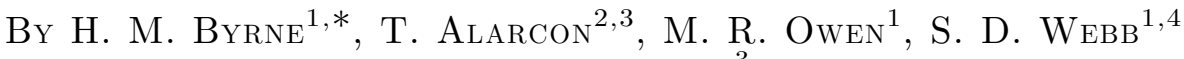 \\ AND P. K. MAIni ${ }^{3}$ \\ ${ }^{1}$ Centre for Mathematical Medicine, School of Mathematical Sciences, \\ University of Nottingham, Nottingham NG7 2RD, UK \\ ${ }^{2}$ Bioinformatics Group, Department of Computer Science, \\ University College London, Gower Street, London WC1E 6BT, UK \\ ${ }^{3}$ Centre for Mathematical Biology, Mathematical Institute, \\ University of Oxford, 24-29 St Giles', Oxford OX1 3LB, UK \\ ${ }^{4}$ Department of Animal and Plant Sciences, University of Sheffield, \\ Western Bank, Sheffield S10 2TN, UK
}

Cancer is a complex disease in which a variety of factors interact over a wide range of spatial and temporal scales with huge datasets relating to the different scales available. However, these data do not always reveal the mechanisms underpinning the observed phenomena. In this paper, we explain why mathematics is a powerful tool for interpreting such data by presenting case studies that illustrate the types of insight that realistic theoretical models of solid tumour growth may yield. These range from discriminating between competing hypotheses for the formation of collagenous capsules associated with benign tumours to predicting the most likely stimulus for protease production in early breast cancer. We will also illustrate the benefits that may result when experimentalists and theoreticians collaborate by considering a novel anti-cancer therapy.

Keywords: solid tumour growth; mathematical modelling; cancer; differential equations

\section{Introduction}

Paradoxically, improvements in healthcare that have enhanced the life expectancy of humans in the Western world have, indirectly, increased the prevalence of certain cancers such as prostate and breast. It remains unclear whether this phenomenon should be attributed to the ageing process or the cumulative effect of prolonged exposure to harmful environmental stimuli, such as ultraviolet light, radiation and carcinogens (Franks \& Teich 1988). Equally, there is compelling evidence that certain genetic abnormalities can predispose individuals to specific cancers (Ilyas et al. 1999).

The variety of factors involved in the development of solid tumours stems, to a large extent, from the fact that 'cancer' is a generic term, often used to

* Author for correspondence (helen.byrne@maths.nottingham.ac.uk).

One contribution of 15 to a Theme Issue 'Biomathematical modelling II'. 
characterize a series of disorders that share common features. At this generic level of description, cancer may be viewed as a cellular disease, in which controls that usually regulate growth and maintain homeostasis are disrupted. Cancer is typically initiated by genetic mutations that lead to enhanced proliferation rate and the formation of an avascular tumour. Since it receives nutrients by diffusion, the size of an avascular tumour is limited to several millimetres in diameter. Further growth relies on the tumour acquiring the ability to stimulate the ingrowth of a new, circulating blood supply from the host vasculature via angiogenesis (Folkman 1974). Once vascularized, the tumour has access to a vast nutrient source and rapid growth ensues. Further, tumour fragments that break away from the primary tumour, on entering the vasculature, may be transported to other organs in which they may establish secondary tumours or metastases that further compromise the host. Invasion is another key feature of solid tumours, whereby contact with the host tissue stimulates the production of enzymes that digest it, liberating space into which the tumour cells migrate. Thus, cancer is a complex, multiscale process. The spatial scales of interest range from the subcellular level to the cellular and macroscopic (or tissue) levels, while the timescales may vary from seconds (or less) for signal transduction pathways to months for tumour doubling times.

In this paper, we review a number of mathematical models that have been developed to describe some of the above aspects of tumour growth. In so doing, we aim to show how mathematical modelling, computation and analysis can generate useful insight into the mechanisms that underpin this devastating disease. With limited space we are unable to explain the variety of mathematical approaches that are being used: we focus on deterministic models, but recommend a variety of references for details of alternative approaches (Preziosi 2003; Mantzaris et al. 2004; Araujo \& McElwain 2004; Komarova 2005).

The remainder of the paper is organized as follows. In $\S 2$, we focus on simple models of angiogenesis and tumour encapsulation, these providing examples of models that were developed after the associated experiments had been performed. In $\S 3$, we consider three-dimensional models of early avascular tumour growth and models of ductal carcinoma in situ (DCIS), focusing on the experimental predictions and questions that such work has stimulated. Section 4 continues with applications where the theoretical and experimental work were combined and illustrates the sort of additional insight that such collaboration can generate. The paper concludes in $\$ 5$ with a brief discussion of the current state-of-the-art in modelling solid tumour growth and of the challenges that lie ahead.

\section{Experiments driving theory}

A facet shared by the models presented in this section is that they were developed after the relevant experiments had been performed and/or hypotheses developed. Even so, they illustrate an important feature that realistic mathematical models should possess: the ability to reproduce data and, in so doing, to provide possible explanations of observed phenomena. We start by reviewing models of tumour angiogenesis (for more details, see Mantzaris et al. 2004 ) and then discuss a model of tumour encapsulation. 


\section{(a) Tumour-induced angiogenesis}

The earliest continuum models of tumour angiogenesis focused on three key physical variables: a generic tumour-derived chemical, termed a tumour angiogenesis factor (TAF), capillary tips and capillary sprouts. The onedimensional model developed by Balding \& McElwain (1985) consisted of three partial differential equations that were derived by applying the principle of mass balance to each species. The capillary tips were assumed to move by chemotaxis towards the TAF source, the tumour, and to emanate from existing vessels or tips at rates that increased with the TAF concentration. Capillary tips were lost as a result of fusion with other tips and vessels (anastomosis). The production of capillary sprouts was assumed to be driven by tip migration, the sprout density increasing at a rate that matched the flux of capillary tips (the 'snail-trail'). The TAF was modelled as a diffusible chemical that was produced by the tumour, underwent natural decay and was consumed by the migrating capillary tips.

Stimulated by more detailed knowledge of the biochemistry involved, Levine et al. (2001) have developed highly complex models that represent an important step towards understanding angiogenesis using biochemical rather than phenomenological arguments. Their models account for interactions between endothelial cells, angiogenic factors and other cell types that are also involved in angiogenesis. The various models exhibit many features of angiogenesis that are observed in vivo (Muthukkaruppan et al. 1982). For example, simulations of successful angiogenesis show an accelerating front of capillary tips and sprouts migrating towards the tumour with the maximum capillary density preceding the maximum capillary sprout density (Byrne \& Chaplain 1995b).

Extending Balding \& McElwain's model to two and three spatial dimensions (Orme \& Chaplain 1997) highlights the shortcomings of using a continuum framework to study angiogenesis. First, angiogenesis is a multi-dimensional process, with tips sprouting in directions other than that of the propagating vascular front. Hence, it is not obvious how the 'snail-trail' approach should be extended to higher spatial dimensions. In addition, the models do not distinguish between different vascular morphologies (a region perfused by one large vessel is equivalent to another region perfused by many small vessels) or between anastomosis (which increases nutrient supply) and capillary tip death (which does not). Finally, vascular remodelling and the impact of blood flow (and haematocrit) on the evolving vasculature are neglected. Only by developing the simple, one-dimensional models of angiogenesis do such problems come to light and stimulate the design of new models that address these deficiencies. Fortunately, several such models are now being developed. For example, Chaplain and co-workers have developed a continuum-discrete model, in which movement of the vessel tips is modelled using a biased random walk (Anderson \& Chaplain 1998), and blood flow through the vessels is included (McDougall et al. 2002).

In spite of their weaknesses, the continuum models provide useful insight into the ways in which different physical mechanisms influence angiogenesis. In particular, the success of angiogenesis is tightly controlled by the balance between endothelial cell proliferation and migration: as the strength of the chemotactic response increases, the tips migrate more rapidly towards the tumour, reducing the time available for tip proliferation, and hence the vascular density when they reach the tumour. 


\section{(b) Tumour encapsulation}

Many benign tumours are surrounded by a rim of collagenous connective tissue, whose presence is an integral determinant of prognosis, and yet the mechanisms by which the capsule forms remain to be elucidated fully. Two complementary theories have been proposed to explain this phenomenon (Barr 1989). The 'Expansive Growth Hypothesis' (EGH) postulates that capsule formation is a passive process: as the tumour expands, it compresses the collagen in the surrounding extracellular matrix (ECM). By contrast, the 'Foreign Body Hypothesis' (FBH) suggests that capsule formation is an active process: the host recognizes the cancer as a foreign body and mounts an immune response by overexpressing collagen. While it is difficult to discriminate between the hypotheses using experimental techniques, mathematical modelling provides a natural framework by which to compare the two alternatives.

Jackson \& Byrne (2002) developed such a model by viewing the tumour-host environment as a mixture of three phases: the cellular phase comprises tumour cells, the ECM phase and the aqueous phase consist primarily of water and dissolved extracellular tissue components. Mass and momentum balance equations are derived for each phase and closed by specifying constitutive relations for various features, including the stresses that develop within the phases and the net rates at which they are produced. The FBH is incorporated by assuming that the ECM production rate is an increasing function of the tumour cell density, with constant of proportionality $\alpha \geq 0$. The EGH is included by assuming that the pressure in the ECM includes a contribution that increases as the tumour cell density increases, with constant of proportionality $\theta \geq 0$. Since species tend to move down pressure gradients, this pressure will drive ECM motion away from regions of high tumour cell density and, thereby, concentrate ECM near the tumour boundary. Numerical simulations of the full system with both hypotheses active confirm that the model can generate a capsule ahead of the invading tumour (see figure 1). The ECM capsule is preserved in simulations having $\alpha=0<\theta$ and disappears when $\theta=0<\alpha$ (results not shown). These results suggest that the EGH provides a more plausible explanation of tumour encapsulation than the FBH.

\section{Theory driving experiments}

Here, we review theoretical models that were developed without relevant data, but are useful nonetheless because they raise questions and yield predictions that stimulate biomedical researchers to design new experiments that will validate (or refute) the theoretical predictions.

\section{(a) The growth of multicellular spheroids}

The majority of models of solid tumour growth are based on mass balance principles for cells and reaction-diffusion equations for growth factors (see Araujo \& McElwain 2004 for a review). The main challenges involved in developing such models are describing how the tumour cells move, divide and die and how the various chemicals diffuse, are produced and absorbed by the tumour cells. In the earliest models (Greenspan 1972; McElwain \& Morris 1978; Adam 1987), 


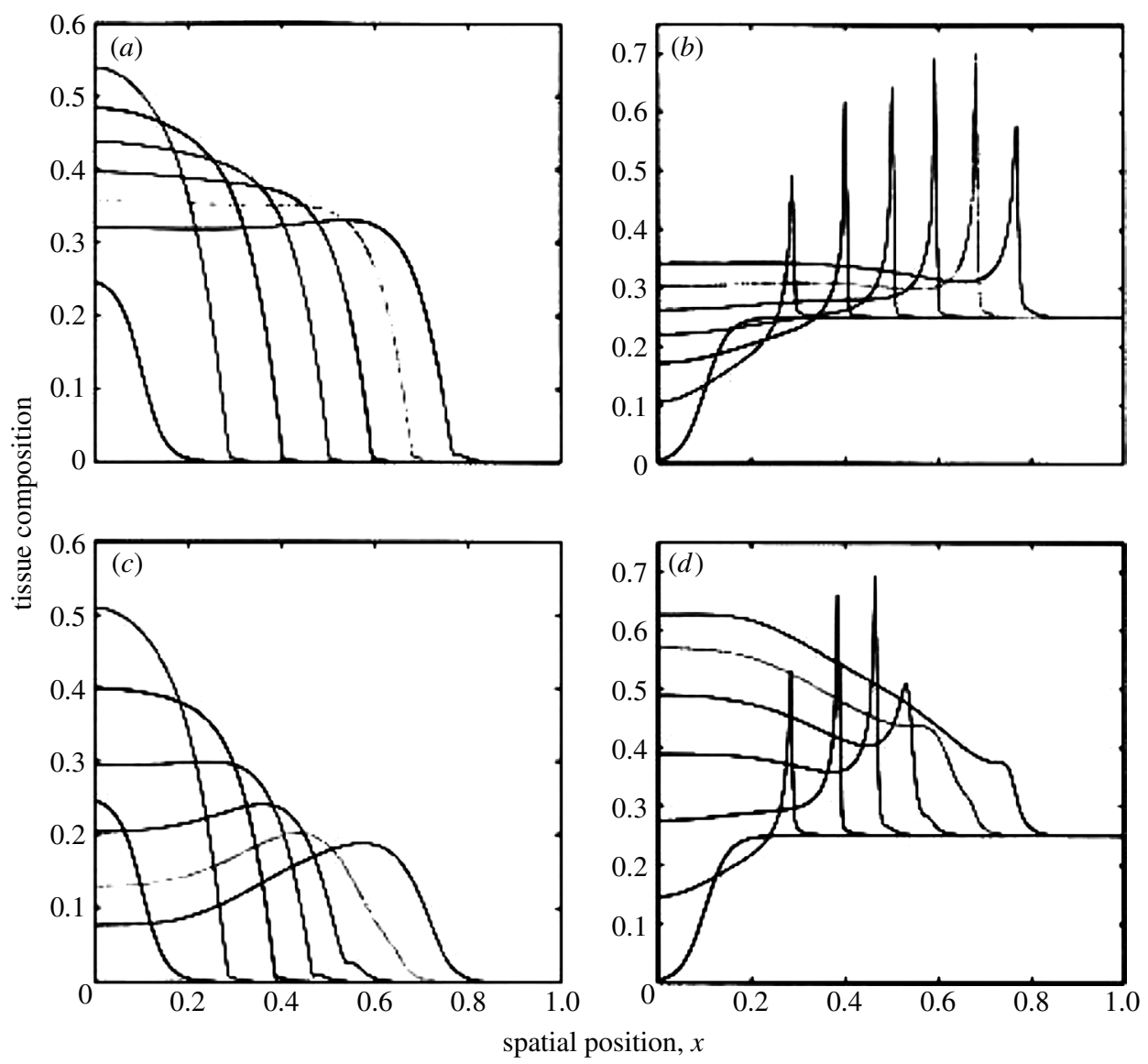

Figure 1. Numerical results showing how the model of tumour encapsulation evolves when both the expansive growth and the foreign body hypotheses are included. The tumour and ECM profiles are plotted at equally spaced time intervals $(t=0,10,20, \ldots, 60$, with $t$ increasing from left to right in all panels) and for two choices of the parameter values: $(a)$ and $(b)$ show the tumour and ECM profiles for the first simulation, while $(c)$ and $(d)$ show the corresponding profiles for the second simulation. In both cases, the tumour invades the ECM $(a$ and $c$ ) and the ECM responds by developing a dense band ahead of the invading tumour front (for details, see Jackson \& Byrne 2002). (Reproduced with permission.)

attention was focused on the one-dimensional growth of a single population of tumour cells of constant density. Mathematical models of this type usually comprise reaction-diffusion equations for the distribution within the tumour of chemicals of interest (e.g. oxygen, glucose or cytotoxic drugs) and an integrodifferential equation characterizing the tumour's volumetric growth rate (Greenspan 1972; McElwain \& Morris 1978; Adam 1987). Such models have enjoyed considerable success, reproducing the multilayered patterns observed in multicellular spheroids cultured in vitro (Folkman \& Hochberg 1973; Sutherland 1988), i.e. an outer rim of nutrient-rich, proliferating cells, a central core of nutrient-starved, necrotic debris and an intermediate region of nutrient-poor, quiescent cells. The models also yield experimentally testable predictions. For example, Byrne \& Chaplain (1998) showed that following the onset of central 
necrosis, the tumour's overall size remains approximately fixed while the size of the necrotic region increases rapidly, a prediction that was confirmed in independent experiments (Groebe \& Mueller-Klieser 1996).

Unfortunately, not all tumours undergo one-dimensional growth and they may be heterogeneous, containing, for example, ECM, immune cells and blood vessels. These observations stimulated demand for three-dimensional models that account for cellular heterogeneity.

Since the early models (Greenspan 1972; McElwain \& Morris 1978) do not generalize readily to higher spatial dimensions, new models were needed (Greenspan 1976; Byrne \& Chaplain 1996). These models introduced the interrelated concepts of cell movement and pressure: pressure gradients, generated by differences in cell proliferation and death, cause cells to move from regions of high cell proliferation and pressure (near the tumour periphery) to regions of net cell death and lower pressure (at the tumour centre). Complete specification of the models necessitated the introduction of constitutive laws to characterize the mechanical properties of the tumour. In the absence of relevant data, Darcy's law was used in both models to relate the cell velocity to the pressure. A key difference between the two models concerns the mechanism that maintains the tumour as a compact mass. Greenspan (1976) invoked surface tension, whereas Byrne \& Chaplain (1998) invoked cell-cell adhesion, assuming that cells on the boundary require additional nutrient to maintain the tumour's compactness and that this would create a jump in the nutrient concentration there. In spite of this difference, both models yielded similar qualitative predictions: if surface tension or cell-cell adhesion is strong enough, then stable, one-dimensional growth occurs. For smaller values of these parameters, one-dimensional growth will not persist and irregular structures, resembling infiltrative invasion, emerge. These results suggest that genetic mutations that weaken the bonds of cell-cell adhesion or reduce surface tension could explain why certain tumours possess highly irregular, fractal-like outer boundaries. Experiments are now needed to establish whether either of the postulated mechanisms is dominant in tumour spheroids.

There is increasing evidence that mechanical effects influence tumour growth. For example, when they embedded tumour spheroids in gels of different stiffnesses, Helmlinger et al. (1997) found that the size to which the spheroids grew decreased as the stiffness of the gel increased. Such results have prompted the development of sophisticated multiphase models (Chen et al. 2001; Jackson \& Byrne 2002; Byrne et al. 2003) and led to predictions including the possible correlation of cell size with stress levels inside the tumour (Roose et al. 2003). When developing these models, consideration of cell-to-cell mechanical interactions is required and constitutive laws such as Darcy's law or Stokes' flow must be employed. Once again, validation of these models requires experimental work to characterize the mechanical behaviour of the different species and how they interact.

\section{(b) Ductal carcinoma in situ}

Ductal carcinoma in situ represents the initial growth stage of breast cancer, during which the tumour is non-invasive, being confined by the duct. The duct is composed of a central lumen, surrounded by a thin layer of epithelial cells, with 
an outer basement membrane (the duct wall) which is made of a network of proteins. DCIS occurs when epithelial cells that have undergone a malignant transformation proliferate. Sustained tumour cell proliferation in the duct increases the outward pressure on the basement membrane. If accompanied by the local production of proteases, this may cause the membrane to yield and enable the tumour cells to invade the tissue (Brummer et al. 1999).

While the literature is now replete with models addressing all aspects of solid tumour growth, little attention has been devoted to DCIS. Xu (2004) studied an existing tumour growth model (Byrne \& Chaplain 1995a) in a radially symmetric, cylindrical geometry, and noted that the spatial patterns (spots, stripes and uniform distributions) exhibited by stationary model solutions were consistent with morphologies commonly observed in DCIS (e.g. cribriform, comedo and papillary). In the paper by Xu (2004), nutrient diffusion limits tumour growth, the duct wall is rigid and tumour properties such as the local cellular density and pressure are neglected. Franks and co-workers developed new models that include these features (Franks et al. 2003, 2004, 2005). The resulting models involve coupling existing models of avascular tumour growth in a cylindrically symmetric tube with mechanical models for the finite deformation of the compliant membrane that confines the tumour, the coupling being mediated by interactions between the expansive forces created by tumour cell proliferation and the stresses that develop in the compliant membrane (Epstein \& Johnston 2001).

The models provide insight into DCIS progression and also generate several hypotheses that could be tested experimentally. For example, the pressure on the duct wall is likely to be greatest at the centre of the tumour and, hence, this is where the duct wall is likely to be breached (Franks et al. 2003). The model is also used to test two hypotheses for the localization, near the duct wall, of proteases that compromise its integrity. Model simulations and asymptotic analysis suggest that the elevated pressure that accompanies tumour growth is more likely to stimulate protease production and localization near the duct wall than hypoxia (Franks et al. 2005).

While several interesting predictions arise from the theoretical work, complementary experiments are now needed to determine the mechanical properties of the duct wall and to validate the predictions. Thus, the models of DCIS illustrate how qualitative insight into a particular system can be generated, even in the absence of relevant data, and how theoretical studies may prompt future experimental work.

\section{Experiments and theory: synergy}

In $\S \S 2$ and 3, the models and experiments were effectively independent, with the experiments preceding the theory $(\$ 2)$ or the theory stimulating new experiments (§3). It is natural to ask whether additional insight can be gained when experiments and theory are combined. Consequently, we now focus on studies which illustrate the benefits that may accrue when experimentalists and theoreticians collaborate. We start by considering anti-angiogenic therapies and then discuss a new therapy involving genetically engineering a patient's macrophages to achieve localized cell killing in certain tumour regions. 


\section{(a) Tumour response to anti-angiogenic treatments}

In the paper by Hahnfeldt et al. (1999), data from tumour-bearing mice treated with a range of angiogenic inhibitors are presented with a theoretical model of tumour growth in response to (tumour-derived) angiogenic stimulators and inhibitors (the drugs). By combining their theoretical and experimental work, Hahnfeldt and co-workers were able to use their model to quantify the effectiveness of different antiangiogenic agents. They were also able to predict conditions under which a tumour will attain a fixed size that is independent of its size at the start of treatment, this being the case when the anti- and proangiogenic factors are in balance. A further advantage of the model developed there is that it could be adapted and applied to a clinical setting.

The above model was extremely simple, involving ordinary differential equations for the tumour mass and the perfusing vasculature (the drug concentration was prescribed and targeted the vasculature directly). Recently, Arakelyan et al. (2005) have developed a complex, multiscale model of vascular tumour growth that accounts for vessel maturation and the regression of immature and mature vessels, phenomena that depend on growth factors, such as VEGF and the angiopoietins and pericytes. Model simulations suggest that rapid tumour growth will occur when the vessel maturation rate is high, due to the rapid increase in vessel density that this causes. By contrast, the model also predicts that lower vessel maturation rates in tumours with lower background vessel densities will create slower growing tumours that possess larger proportions of immature vessels and may exhibit oscillatory growth dynamics (when the immature vessels regress, perfusion is limited and a new cycle of angiogenesis stimulated). Experimental results confirm the model predictions and, used in combination with the mathematical model, strengthen the hypothesis that variations in tumour growth rates may be strongly influenced by the strength of the angiogenic response elicited in the tumour environment.

\section{(b) Novel anti-cancer therapies}

Increased understanding of how different solid tumours grow means that it is now possible to design patient-specific treatment protocols. Even so, certain tumour sites, in particular hypoxic regions, remain notoriously difficult to target with existing therapies. Hypoxic regions are usually furthest from the blood vessels that perfuse the tissue, hampering drug delivery. Furthermore, tumour cells respond to hypoxia by slowing their rate of proliferation, so that antiproliferative therapies will have minimal effect there. Hypoxic tumour cells are also potent sources of angiogenic factors such as VEGF.

A possible solution to this problem is linked to the observation that macrophages localize in hypoxic regions of many tumours, particularly breast. A new therapy using genetically engineered macrophages exploits their tendency, when reinjected into the patient, to migrate preferentially to low oxygen regions where they are activated to release anti-tumour drugs. Particularly, the low oxygen levels that characterize solid tumours are peculiar to tumours and not usually observed in other organs. Hence, the therapy promises increased tumour specificity, with reduced side-effects.

Preliminary results are promising, showing up to $30 \%$ reduction in the size of avascular tumours treated with appropriately engineered macrophages (Griffiths 


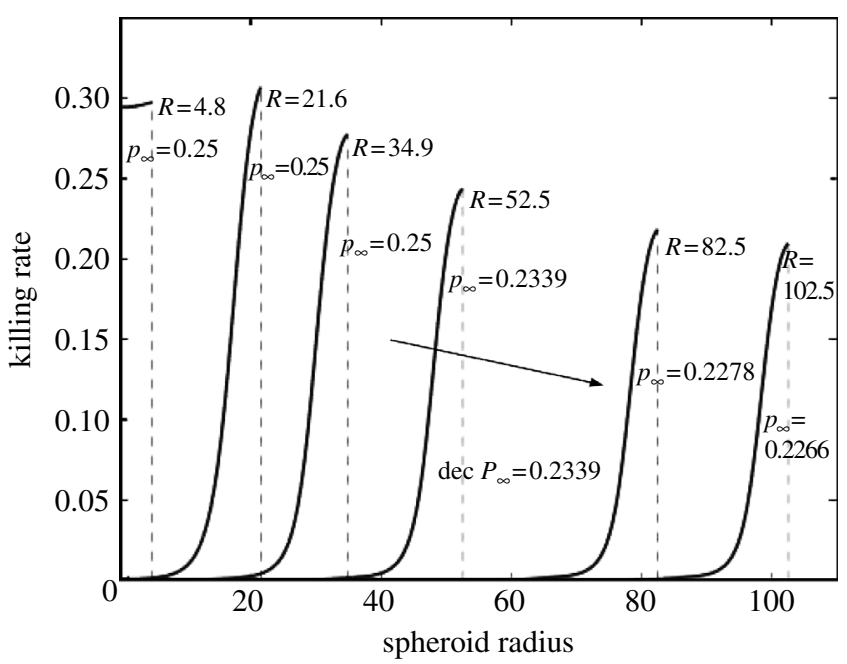

Figure 2. Numerical simulations showing where cell killing occurs within spheroids treated with engineered macrophages, the different curves corresponding to different levels of drug cytotoxicity (as $p_{\infty}$ increases the drug is more toxic to the tumour cells). In each case, cell death is localized near to the tumour periphery, where cell proliferation is greatest, yielding a response similar to that for conventional chemotherapy (for details, see Webb et al. submitted).

et al. 2000). Many questions remain to be answered before this approach can be used routinely. Of particular interest is establishing where cell kill occurs when engineered macrophages are used. In order to address this issue, models of macrophage infiltration into tumour spheroids growing either in vitro (in free suspension) or in vivo (in healthy tissue) have been developed (Owen et al. 2004; Webb et al. submitted). In these models, it is important to describe precisely how the macrophages exert their influence. In one approach currently under investigation, the macrophages produce an enzyme under hypoxia. The enzyme converts to active form a pro-drug, which is injected separately. When these features are incorporated into models of spheroid growth in vitro, cell kill is localized towards the tumour periphery, where the proliferation rate is highest (see figure 2). Since a similar response would result on application of a conventional drug that targets proliferating cells, it is natural to question the value of such an elaborate treatment. Fortunately, results from in vivo simulations indicate that the engineered macrophages may be beneficial when used to treat patients, as they predict reduced toxicity (see figures 3 and 4).

The benefit of combining the experimental and theoretical endeavours should now be clear: on the basis of the in vitro results alone, the macrophage therapy might be dismissed since it appears to act in the same way as conventional therapies. However, the models show that in vivo the therapy has significant benefits, in terms of enhanced tumour specificity and weaker side-effects.

\section{Conclusions}

The variety of phenomena involved in cancer, the range of spatial and temporal scales over which they act and the complex way in which they are inter-related 

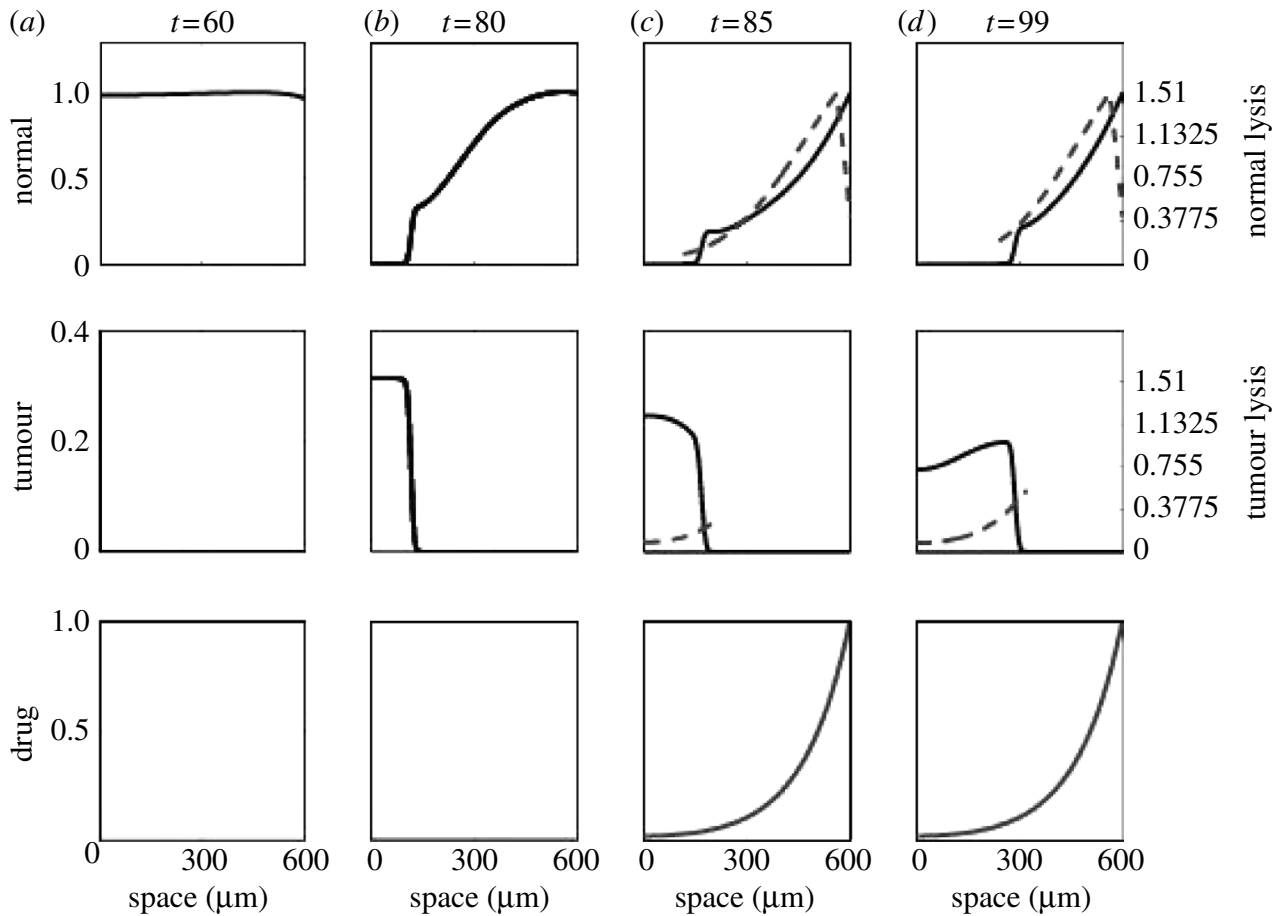

Figure 3. Series of profiles showing how a region of healthy (avascular) tissue in which a tumour has been implanted responds to conventional chemotherapy that is delivered to the region from a blood vessel located on the right-hand side of the spatial domain (for details, see Webb et al. submitted). Since the drug targets proliferating cells, it destroys much of the healthy tissue before reaching the tumour site, enabling the tumour to invade unhindered through the region. Solid lines represent cell densities, dashed lines represent cell death rates.

mean that it is practically impossible to deduce how the different mechanisms interact by intuition alone. Through the development of well-founded theoretical models of solid tumour growth, such as those reviewed in this article, we believe that mathematics, in conjunction with related experimental work, can be a valuable tool with which to address such questions.

There is now a large literature devoted to modelling solid tumour growth. Even so, many issues remain to be resolved. For example, existing models tend to focus on generic tumours and relatively few are specialized to specific tumours such as gliomas (Swanson et al. 2003) or DCIS (Franks et al. 2005). Further, existing models typically focus on a single spatial scale. As a result, they are unable to address the fundamental problem of how phenomena at different scales are coupled or to combine, in a systematic manner, data from the various scales (Alarcon et al. 2004a; Arakelyan et al. 2005). Alarcon et al. (2004a) formulated a hybrid cellular automaton that contains interlinked elements that describe processes at each spatial scale: progress through the cell cycle (Alarcon et al. $2004 b$ ) and the production of angiogenesis factors are accounted for at the subcellular level; cell-cell interactions are treated at the cellular level; and, at the tissue scale, attention focuses on the vascular network, whose structure adapts in response to blood flow and angiogenic factors produced at the subcellular level. 


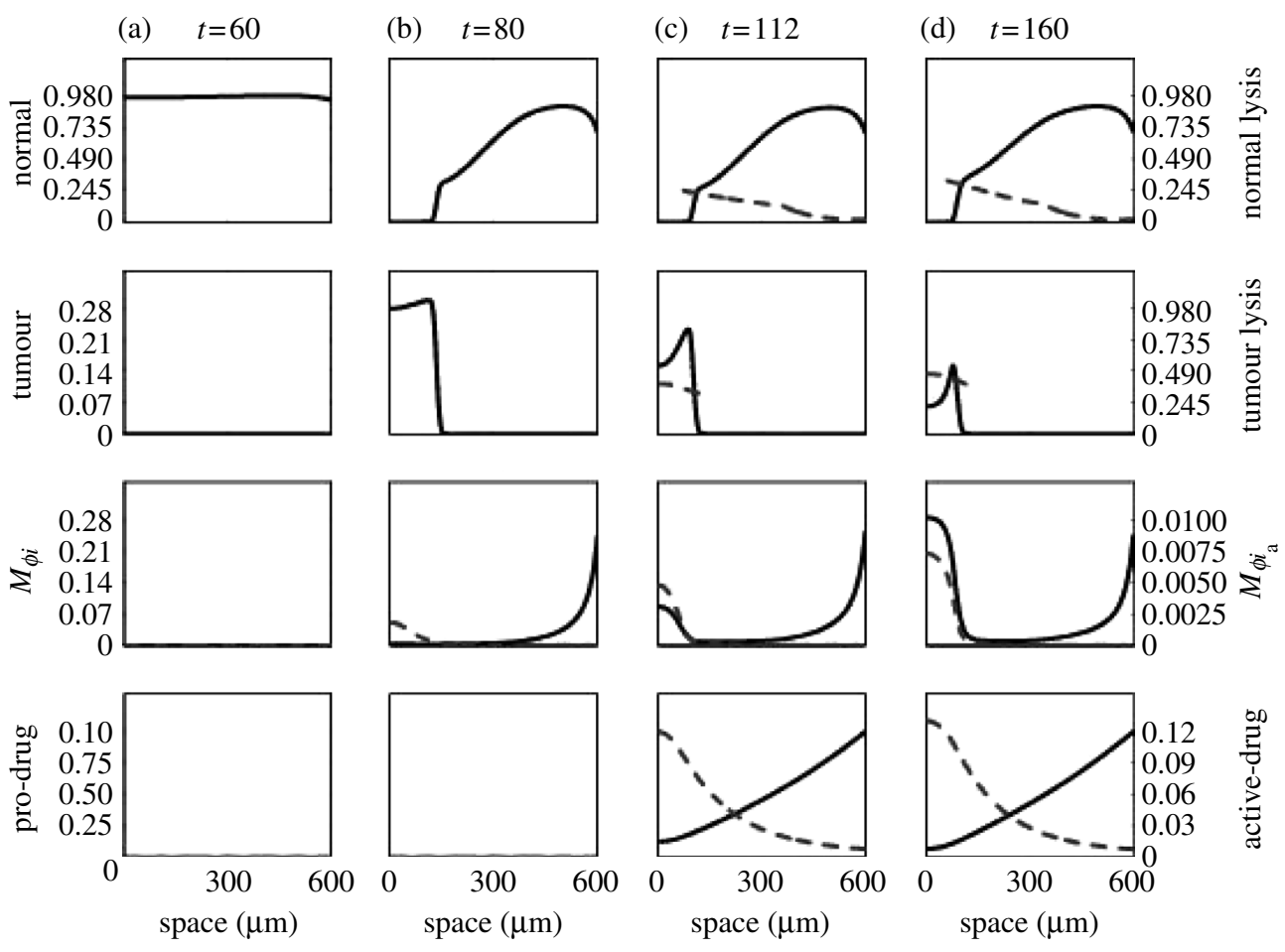

Figure 4. Series of profiles showing how the same region of tissue as in figure 3 responds to treatment with the engineered macrophages (for details, see Webb et al. submitted). In this case, active drug is produced in hypoxic tumour regions and preferentially targets tumour cells at the leading front, reducing the damage to the healthy tissue. Solid lines represent cell densities, dashed lines represent cell death rates.

Further coupling between the different spatial scales arises from the transport of blood-borne oxygen into the tissue and its uptake at the cellular level. The model is undoubtedly complex and yields many predictions that warrant experimental validation. For example, the simulations presented in figure 5 indicate that an accurate description of blood flow and, hence, oxygen delivery to the tumour is important if realistic models of this form are to be developed: if oxygen is distributed uniformly through the vasculature, the tumour grows as a compact mass, expanding radially outwards. By contrast, when oxygen is distributed heterogeneously through the vasculature, the tumour's growth is localized in regions of high oxygen.

Multiscale modelling is a huge challenge for mathematicians. Given that it is now recognized that the traditional 'top-down' approach to modelling in the life sciences does not make full use of the huge amount of data being generated experimentally, there is a move to model 'bottom-up'. However, here we have to be careful that we do not simply replace the biological system we are trying to understand by a huge computational model that we have no chance of ever understanding! One way forward is to develop 'toy' models at one level that are then used as input at another level. For example, if we wish to understand how regulation of the HIF- $1 \alpha$ pathway leads to tissue growth under hypoxic conditions, we need first to write down the detailed biochemical pathways 
(a)

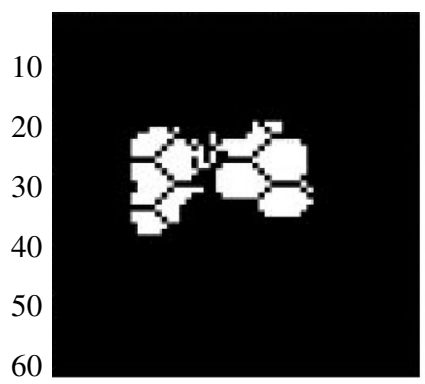

$(d)$

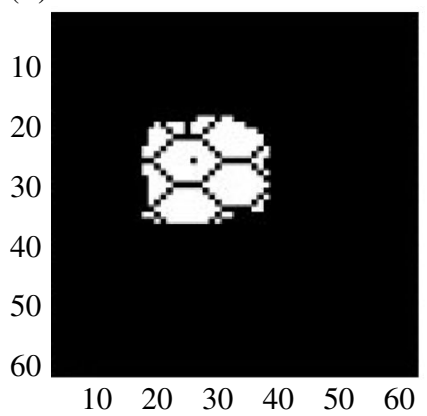

(b)

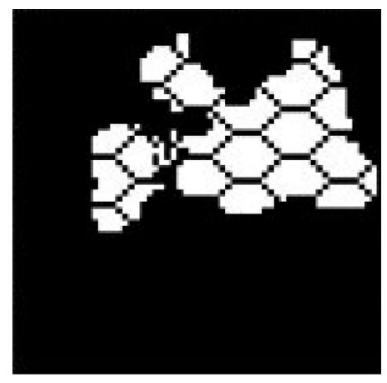

$(e)$

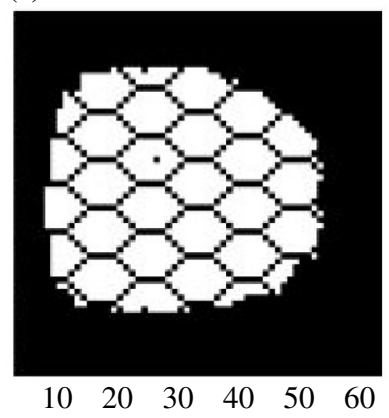

(c)

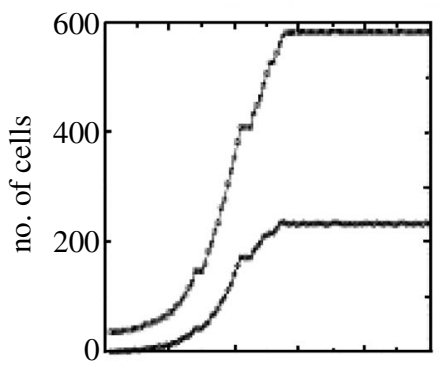

$(f)$

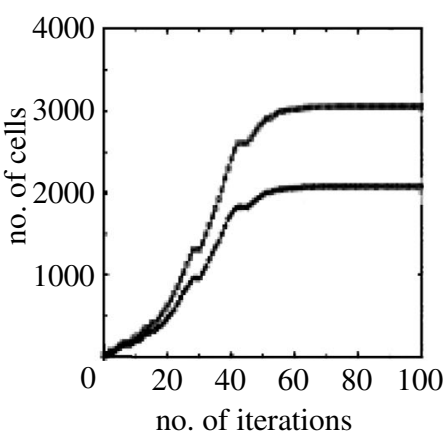

Figure 5. Series of profiles showing how the pattern of tumour invasion depends on the way in which the vessels deliver oxygen to the tissue (for details, see Alarcon et al. 2004a). When the oxygen is distributed uniformly through the vasculature (lower figures), the tumour grows as a compact, almost circular mass and eventually colonizes the entire tissue. When the oxygen is distributed heterogeneously (upper figures), the tumour's growth is more irregular and confined to those regions of the tissue where oxygen levels are highest. In panels $(a),(b),(d)$ and $(e)$, white spaces represent tumour cells and black spaces are either empty or occupied by vessels. Panels $(c)$ and $(f)$ show the time evolution of the number of cancer cells for non-uniform and uniform oxygen distribution, respectively. The upper curves (squares) represent the total number of cancer cells (proliferating and quiescent) and the lower curves (diamonds) represent the number of quiescent tumour cells. In both simulations, the normal cells are rapidly eliminated by the tumour population, and therefore do not appear. (Reproduced with permission.)

regulating $\mathrm{HIF}$, understand them and then derive, using, for example, multitimescale approaches and coarse-graining, a simple model which can be linked to a model for how HIF upregulates VEGF production and hence angiogenesis, and how this increases nutrient delivery leading to increased tissue growth. The detailed modelling at the biochemical level will allow us to address issues, such as which pathway might be the key one to attack from a therapeutic point of view, but will have limited value in a whole organ model. Thus, we really need to develop a suite of models which are used to answer different questions. Of course, a key problem here will be to ensure that in simplifying the model we still capture all the salient features. This will require using intra- as well as interdisciplinary research approaches, for example, using perturbation analysis of ordinary differential equations, and Boolean network or topological approaches, to make sure that we fully understand the regulatory network being studied. 
Mathematical and computational modelling have a number of roles to play in cancer research, and we have chosen in this review to focus on certain models which illustrate each of these roles. For example, the first model that anyone develops (usually the experimentalist) is a 'word' model, that is, the assumption that a limited set of processes combine to produce an observed phenomenon. The verbal reasoning used to manipulate such word models is necessarily linear. However, most biological interactions are nonlinear, and the natural medium for manipulating such ideas is mathematics. Therefore, one role of a mathematical model is to verify hypotheses and we have given a number of examples of this in the present paper. Modelling can also help to develop our intuition and to understand what appear to be counter-intuitive results.

Other roles of modelling are to generate hypotheses and to make predictions, and, again, we have illustrated a number of such examples above. However, this is as far as a model can go. There must be an iteration between modelling and experiment, where theoreticians develop models based on in-depth discussion with experimentalists, who, in turn, test the predictions of the model. Based on these results, the model is refined and the iteration continues, allowing us hopefully to converge to a full understanding of the phenomenon at hand. This is an example of the type of interdisciplinary research discussed in the paper by Welsh et al. (2006). However, a disappointing aspect of cancer research thus far has been the lack of experimental testing of model predictions (Komarova 2005). This arises mainly because the groups doing theory and those doing experiment are not embedded with each other. This was one of the prime motivations for us to develop the Integrative Biology programme (discussed in the paper by Welsh et al. (2006), and in which both Byrne and Maini are co-investigators) directly to address this problem. The benefits of such an interaction are very apparent. In 1996, Gatenby and Gawlinski developed a mathematical model for the acidmediated invasion hypothesis for tumour spread. The model predicted that under certain circumstances a gap would arise between the advancing tumour front and the regressing normal tissue (Gatenby \& Gawlinski 1996). As Gatenby is himself a clinician, he was able to test this prediction and show it to be true. Unfortunately, few individuals have access to both the theoretical know-how and the experimental equipment to work in this way, and once again this highlights the need for a new way to do science (Komarova 2005; Welsh et al. 2006).

In conclusion, in this article we have explained why we believe theoretical modelling has an important role to play in advancing our understanding of solid tumour growth in particular and medicine in general. We hope that in the future, as these benefits become more widely recognized, mathematicians will be routinely involved in the design and analysis of experimental work.

\section{Editors' note}

Please see also related communications in this focussed issue by Pinter \& Shohet (2006) and Schaller \& Meyer-Hermann (2006).

The authors gratefully acknowledge support from the EU Research Training Network (5th Framework): 'Using mathematical modelling and computer simulation to improve cancer therapy' (TA) and the EPSRC (via an advanced research fellowship, HMB). 


\section{References}

Adam, J. A. 1987 A mathematical model of tumour growth. II. Effects of geometry and spatial uniformity on stability. Math. Biosci. 86, 183-211. (doi:10.1016/0025-5564(87)90010-1)

Alarcon, T., Byrne, H. M. \& Maini, P. K. 2004a Towards whole organ modelling of tumour growth. Prog. Biophys. Mol. Biol. 85, 451-472. (doi:10.1016/j.pbiomolbio.2004.02.004)

Alarcon, T., Byrne, H. M. \& Maini, P. K. $2004 b$ A mathematical model of the effects of hypoxia on the cell-cycle of normal and cancer cells. J. Theor. Biol. 229, 395-411. (doi:10.1016/j.jtbi.2004. 04.016)

Anderson, A. R. A. \& Chaplain, M. A. J. 1998 Continuous and discrete mathematical models of tumour-induced angiogenesis. Bull. Math. Biol. 60, 857-899. (doi:10.1006/bulm.1998.0042)

Arakelyan, L., Merbl, Y. \& Agur, Z. 2005 Vessel maturation effects on tumour growth: validation of a computer model in implanted human ovarian carcinoma spheroids. Eur. J. Cancer 41, 159-167. (doi:10.1016/j.ejca.2004.09.012)

Araujo, R. P. \& McElwain, D. L. S. 2004 A history of the study of solid tumour growth: the contribution of mathematical modelling. Bull. Math. Biol. 66, 1039-1091. (doi:10.1016/j.bulm. 2003.11.002)

Balding, D. \& McElwain, D. L. S. 1985 A mathematical model of tumour-induced capillary growth. J. Theor. Biol. 114, 53-73.

Barr, L. C. 1989 The encapsulation of tumours. Clin. Exp. Metastasis 7, 277-282. (doi:10.1007/ BF01753680)

Brummer, O., Athar, S., Riethdorf, L., Loning, T. \& Herbst, H. 1999 Matrix-metalloproteinases 1, 2, and 3 and their tissue inhibitors 1 and 2 in benign and malignant breast lesions: an in situ hybridization study. Virchows Arch. 435, 566-573. (doi:10.1007/s004280050442)

Byrne, H. M. \& Chaplain, M. A. J. 1995a Growth of nonnecrotic tumours in the presence and absence of inhibitors. Math. Biosci. 130, 151-181. (doi:10.1016/0025-5564(94)00117-3)

Byrne, H. M. \& Chaplain, M. A. J. $1995 b$ Mathematical models for tumour angiogenesis: numerical simulations and nonlinear wave solutions. Bull. Math. Biol. 57, 461-486. (doi:10.1016/S00928240(05)81778-1)

Byrne, H. M. \& Chaplain, M. A. J. 1996 Modelling the role of cell-cell adhesion in the growth and development of carcinomas. Math. Comput. Modell. 24, 1-17. (doi:10.1016/S08957177(96)00174-4)

Byrne, H. M. \& Chaplain, M. A. J. 1998 Necrosis and apoptosis: distinct cell loss mechanisms in a mathematical model of avascular tumour growth? J. Theor. Med. 1, 223-236.

Byrne, H. M., King, J. R., McElwain, D. L. S. \& Preziosi, L. 2003 A two-phase model of solid tumour growth. Appl. Math. Lett. 16, 567-573. (doi:10.1016/S0893-9659(03)00038-7)

Chen, C. Y., Byrne, H. M. \& King, J. R. 2001 The influence of growth-induced stress from the surrounding medium on the development of multicell spheroids. J. Math. Biol. 43, 191-220. (doi:10.1007/s002850100091)

Epstein, M. \& Johnston, C. R. 2001 On the exact speed and amplitude of solitary waves in fluidfilled elastic tubes. Proc. R. Soc. A 457, 1195-1213. (doi:10.1098/rspa.2000.0715)

Folkman, J. 1974 Tumour angiogenesis. Adv. Cancer Res. 19, 331-358.

Folkman, J. \& Hochberg, M. 1973 Self-regulation of growth in three-dimensions. J. Exp. Med. 138, 745-753. (doi:10.1084/jem.138.4.745)

Franks, L. M. \& Teich, N. 1988 Introduction to the cellular and molecular biology of cancer. Oxford, UK: Oxford University Press.

Franks, S. J., Byrne, H. M., King, J. R. \& Lewis, C. E. 2003 Modelling the early growth of ductal carcinoma in situ. J. Math. Biol. 47, 424-452. (doi:10.1007/s00285-003-0214-x)

Franks, S. J., Byrne, H. M., Mudhar, H. S., Underwood, J. C. E. \& Lewis, C. E. 2004 Mathematical modelling of comedo ductal carcinoma in situ of the breast. Math. Med. Biol. 20, 277-308.

Franks, S. J., Byrne, H. M., Underwood, J. C. E. \& Lewis, C. E. 2005 Biological inferences from a mathematical model of comedo duct carcinoma in situ of the breast. J. Theor. Biol. 232, 523-543. (doi:10.1016/j.jtbi.2004.08.032) 
Gatenby, R. A. \& Gawlinski, E. T. 1996 A reaction-diffusion model of cancer invasion. Cancer Res. 56, 5745-5753.

Greenspan, H. P. 1972 Models for the growth of a solid tumour by diffusion. Stud. Appl. Math. 52, $317-340$.

Greenspan, H. P. 1976 On the growth and stability of cell cultures and solid tumours. J. Theor. Biol. 56, 229-242.

Griffiths, L., Binley, K., Iqball, S., Kan, O., Maxwell, P., Lewis, C. E., Harris, A. L., Kingsman, S. \& Naylor, S. 2000 The macrophage - a novel system to deliver gene therapy to pathological hypoxia. Gene Ther. 7, 255-262. (doi:10.1038/sj.gt.3301058)

Groebe, K. \& Mueller-Klieser, W. 1996 On the relation between size of necrosis and diameter of tumour spheroids. Int. J. Radiat. Oncol. 34, 395-401. (doi:10.1016/0360-3016(95)02065-9)

Hahnfeldt, P., Panigraphy, D., Folkmanm, J. \& Hlatky, L. 1999 Tumour development under angiogenic signalling: a dynamical theory of tumour growth, treatment response and postvascular dormancy. Cancer Res. 59, 4770-4775.

Helmlinger, G., Netti, P. A., Lichtenbeld, H. C., Melder, R. J. \& Jain, R. K. 1997 Solid stress inhibits the growth of multicellular tumour spheroids. Nat. Biotechnol. 15, 778-783. (doi:10. 1038/nbt0897-778)

Ilyas, M., Straub, J., Tomlinson, I. P. \& Bodmer, W. F. 1999 Genetic pathways in colorectal and other cancers. Eur. J. Cancer 35, 1986-2002. (doi:10.1016/S0959-8049(98)00431-6)

Jackson, T. L. \& Byrne, H. M. 2002 A mathematical model of tumour encapsulation. Math. Biosci. 180, 307-328.

Komarova, N. L. 2005 Mathematical modelling of tumorigenesis: mission impossible. Curr. Opin. Oncol. 17, 39-43. (doi:10.1097/01.cco.0000143681.37692.32)

Levine, H., Pamuk, S., Sleeman, B. D. \& Nilsen-Hamilton, M. 2001 Mathematical modelling of capillary formation and development in tumour angiogenesis: penetration into the stroma. Bull. Math. Biol. 63, 801-863. (doi:10.1006/bulm.2001.0240)

Mantzaris, N., Webb, S. \& Othmer, H. G. 2004 Mathematical modelling of tumour-induced angiogenesis. J. Math. Biol. 49, 111-187. (doi:10.1007/s00285-003-0262-2)

McDougall, S. R., Anderson, A. R. A., Chaplain, M. A. J. \& Sherratt, J. A. 2002 Mathematical modelling of flow through vascular networks: implications for tumour-induced angiogenesis and chemotherapy strategies. Bull. Math. Biol. 64, 673-702. (doi:10.1006/bulm.2002.0293)

McElwain, D. L. S. \& Morris, L. E. 1978 Apoptosis as a volume loss mechanism in mathematical models of solid tumour growth. Math. Biosci. 39, 147-157. (doi:10.1016/00255564(78)90033-0)

Muthukkaruppan, V. R., Kubai, L. \& Auerbach, R. 1982 Tumour-induced neovascularisation in the mouse eye. J. Natl Cancer Inst. 69, 699-705.

Orme, M. E. \& Chaplain, M. A. J. 1997 Two-dimensional models of tumour angiogenesis and antiangiogenesis strategies. IMA J. Math. Appl. Med. Biol. 14, 189-205.

Owen, M. R., Byrne, H. M. \& Lewis, C. E. 2004 Mathematical modelling of the use of macrophages as vehicles for drug delivery to hypoxic tumour sites. J. Theor. Biol. 226, 377-391. (doi:10.1016/ j.jtbi.2003.09.004)

Pinter, G. G. \& Shohet, J. L. 2006 Two fluid compartments in the renal inner medulla: a view through the keyhole of the concentrating process. Phil. Trans. R. Soc. A 364, 1551-1561. (doi:10.1098/rsta.2006.1774)

Preziosi, L. 2003 Cancer modelling and simulation. London: CRC Press.

Roose, T., Netti, P. A., Munn, L. L., Boucher, Y. \& Jain, R. K. 2003 Solid stress generated by spheroid growth estimated using a linear poroelasticity model. Microvasc. Res. 66, 204-212. (doi:10.1016/S0026-2862(03)00057-8)

Schaller, G. \& Meyer-Hermann, M. 2006 Continuum versus discrete model: a comparison for multicellular tumour spheroids. Phil. Trans. R. Soc. A 364, 1443-1464. (doi:10.1098/rsta.2006. 1780)

Sutherland, R. M. 1988 Cell and environment interactions in tumour microregions: the multicell spheroid model. Science 240, 177-184. 
Swanson, K. R., Bridge, C., Murray, J. D. \& Ellsworthy, C. A. 2003 Virtual and real brain tumours: using mathematical modelling to quantify glioma growth and invasion. J. Neurol. Sci. 216, 1-10. (doi:10.1016/j.jns.2003.06.001)

Webb, S. D., Owen, M. R., Byrne, H. M., Murdoch, C. \& Lewis, C. E. Submitted. A new macrophage-based gene therapy for cancer: experimental and theoretical investigations.

Welsh, E., Jirotka, M. \& Gavaghan, D. 2006 Post-genomic science: multidisciplinary and largescale collaborative research and its organisational and technological implications for the science research process. Phil. Trans. R. Soc. A 364, 1533-1549. (doi:10.1098/rsta.2006.1785)

Xu, Y. 2004 A free boundary problem model of ductal carcinoma in situ. Discrete Cont. Dyn. Syst. 4, 337-348. 